\title{
A Single Amino Acid Change in Nramp6 from Sedum Alfredii Hance Affects Cadmium Accumulation
}

\author{
Zhuchou Lu ${ }^{1,2}{ }^{-}$, Shuangshuang Chen ${ }^{1,2,3}$, Xiaojiao Han ${ }^{1,2}$, Jin Zhang ${ }^{4}$, Guirong Qiao ${ }^{1,2}$, \\ Yugen Jiang ${ }^{5}$, Renying Zhuo ${ }^{1,2, * \mathbb{B}}$ and Wenmin Qiu ${ }^{1,2, *}$ \\ 1 State Key Laboratory of Tree Genetics and Breeding, Chinese Academy of Forestry, Xiangshan Road, \\ Beijing 100091, China; luzc9581@163.com (Z.L.); chenshuang@jaas.ac.cn (S.C.); \\ hanxiaojiao1004@163.com (X.H.); gr_q1982@163.com (G.Q.) \\ 2 The Research Institute of Subtropical Forestry, Chinese Academy of Forestry, Fuyang, \\ Hangzhou 311400, China \\ 3 Institute of Leisure Agriculture, Jiangsu Academy of Agriculture Sciences, Nanjing 210014, China \\ 4 Biosciences Division, Oak Ridge National Laboratory, Oak Ridge, TN 37831, USA; zhangj1@ornl.gov \\ 5 Agricultural Technology Extension Center of Fuyang District, Hangzhou 311400, China; fuyangjyg@163.com \\ * Correspondence: zhuory@gmail.com (R.Z.); qiuwenmin05@gmail.com (W.Q.)
}

Received: 9 April 2020; Accepted: 26 April 2020; Published: 30 April 2020

\begin{abstract}
SaNramp6 in Sedum alfredii encodes a membrane-localized metal transporter. We isolated the SaNramp6h allele from the hyperaccumulating ecotype (HE) of S. alfredii. When this allele was expressed in transgenic yeast and Arabidopsis thaliana, it enhanced their cadmium (Cd) sensitivity by increased $\mathrm{Cd}$ transport and accumulation. We isolated another allele, SaNramp6n, from a nonhyperaccumulating ecotype (NHE) of $S$. alfredii. Amino acid sequence comparisons revealed three amino acid differences between SaNramp6h and SaNramp6n. We investigated the Cd transport activity of the Nramp6 allele, and determined which residues are essential for the transport activity. We conducted structure-function analyses of SaNramp6 based on site-directed mutagenesis and functional assays of the mutants in yeast and Arabidopsis. The three residues that differed between SaNramp6h and SaNramp6n were mutated. Only the L157P mutation of SaNramp6h impaired Cd transport. The other mutations, S218N and T504A, did not affect the transport activity of SaNramp6h, indicating that these residues are not essential for metal selectivity. Transgenic plants overexpressing SaNramp $6 h^{\mathrm{L} 157 \mathrm{P}}$ showed altered metal accumulation in shoots and roots. Our results suggest that the conserved site L157 is essential for the high metal transport activity of SaNramp6h. This information may be useful for limiting or increasing $\mathrm{Cd}$ transport by other plant natural resistance associated macrophage protein (NRAMP) proteins.
\end{abstract}

Keywords: Nramp6; Cadmium accumulation; site-directed mutagenesis; Sedum alfredii Hance

\section{Introduction}

Cadmium (Cd) is a heavy metal with strong biological toxicity that is widely distributed in soil, water and the atmosphere. It causes great harm to animals and plants and can threaten human health [1]. Contamination of soil and water with $\mathrm{Cd}$ represents a major environmental problem [2]. Hyperaccumulator plants can grow in heavy metal-contaminated areas and accumulate high concentrations of heavy metals in their aboveground parts without toxicity symptoms [3]. Hyperaccumulator plants have potential applications in phytoremediation. Compared with nonaccumulator plants, hyperaccumulators show enhancement of one or more physiological mechanisms; for example, heavy metal uptake, root-to-shoot translocation, detoxification, and/or sequestration [4]. 
Plants respond to heavy metal stress by altering diverse genetic pathways. Such pathways include the synthesis of signaling molecules and stress-related proteins, such as antioxidant enzymes, metal chelators, osmotic regulators, and heavy metal transporters. These proteins function in the absorption, transport, intracellular transport, and detoxification of heavy metals, which are necessary for plants to maintain intracellular metal homeostasis. It is of great theoretical significance and practical value to study the physiological response of plants to $\mathrm{Cd}$ stress and the molecular mechanism of related genes and stress signal transduction pathways.

Heavy metal transporters play important roles in metal ion homeostasis, and include the cation diffusion facilitator (CDF) family [5], the heavy metal ATPase (HMA) family [6], the yellow-stripe 1-like (YSL) family [7,8], the ATP-binding cassette (ABC) family [9], the multidrug and toxic compound extrusion (MATE) family [10], the cation/H+ exchanger (CAX) family [11], zinc $(\mathrm{Zn})$-regulated transporters, the iron (Fe)-regulated transporter-like protein (ZIP) family [12], and the natural resistance associated macrophage protein (NRAMP) family [13]. The NRAMP family of proteins is evolutionarily conserved and functions as a symporter to transport diverse metals including iron $(\mathrm{Fe}), \mathrm{Zn}$, manganese $(\mathrm{Mn})$, and $\mathrm{Cd}$. Most of our knowledge about the mechanisms of these transporters is derived from studies on Arabidopsis thaliana and rice (Oryza sativa), whose NRAMPs play critical roles in Mn homeostasis and Cd toxicity [13-18]. Six NRAMP gene family members have been identified in Arabidopsis. Their encoded proteins include AtNRAMP1, which transports Mn, $\mathrm{Fe}$, and Cd [15]; AtNRAMP3 and AtNRAMP4, which transport Mn and Fe [16]; and AtNRAMP6, which transports $\mathrm{Cd}$ [17]. In rice, OsNRAMP5 is a constitutively expressed protein essential for Fe, $\mathrm{Mn}$, and Cd uptake [13], and knockout of OsNramp5 was shown to reduce Cd accumulation in rice [18].

Previously, Zhang et al. (2015) identified five NRAMP genes from the transcriptome of the hyperaccumulating ecotype (HE) Sedum alfredii [19]. Among them, Nramp6 encodes cell membrane-localized protein that is highly expressed in the shoot, where it plays a role in transporting $\mathrm{Cd}$ and Mn $[19,20]$. Transgenic A. thaliana expressing SaNramp6h exhibited high Cd accumulation levels. However, the structure-function relationship of SaNramp6h remains elusive to date. A previous study identified two S. alfredii genotypes, one of which was growing in the Southeast of China in an abandoned mine region with a high concentration of heavy metals in the soil [21]. S. alfredii (Crasulaceae) is the only known Cd-hyperaccumulating species that is not in the Brassica family [22]. The $\mathrm{Cd}$ concentrations in the leaves and stems of the hyperaccumulating ecotype (HE) reached 9000 and $6500 \mathrm{mg} \mathrm{kg}^{-1}$ (DW), respectively, when plants were supplied with $400 \mu \mathrm{mol} \mathrm{Cd} \mathrm{L}{ }^{-1}$ [23]; the nonhyperaccumulating ecotype (NHE) is not tolerant to heavy metals, and its leaf Cd concentrations reached only $533 \mathrm{mg} / \mathrm{kg}$ [24]. The reason for this difference is unknown.

In this study, we identified and analyzed SaNramp6h from the HE of S. alfredii and its homolog SaNramp6n from the NHE of $S$. alfredii. A sequence comparison analysis revealed three amino acid differences between SaNramp6h and SaNramp6n-Leu157Pro, Ser218Asn and Thr504Ala. We speculated that these differences in this homologous gene may be some of the reasons for the difference in $\mathrm{Cd}$ tolerance between the two genotypes. Each of the three amino acid residues that differ between SaNramp6h and SaNramp6n was altered by PCR site-directed mutation, and the effects of these mutations on $\mathrm{Cd}$ uptake and accumulation were test by expression in yeast and $A$. thaliana.

\section{Results}

\subsection{Sequence Alignment and Expression Analyses of SaNramp6h and SaNramp6n}

The coding regions of SaNramp6 from the two distinct ecotypes studied previously were sequenced and compared. This comparison showed that five nucleotides differ between SaNramp6h and SaNramp6n (Figure 1), corresponding to three amino acid differences between SaNramp6h and SaNramp6n at position 157 (Leu to Pro), 218 (Ser to Asn), and 504 (Thr to Ala). The difference at position 119 and 176 resulted from synonymous mutation. 


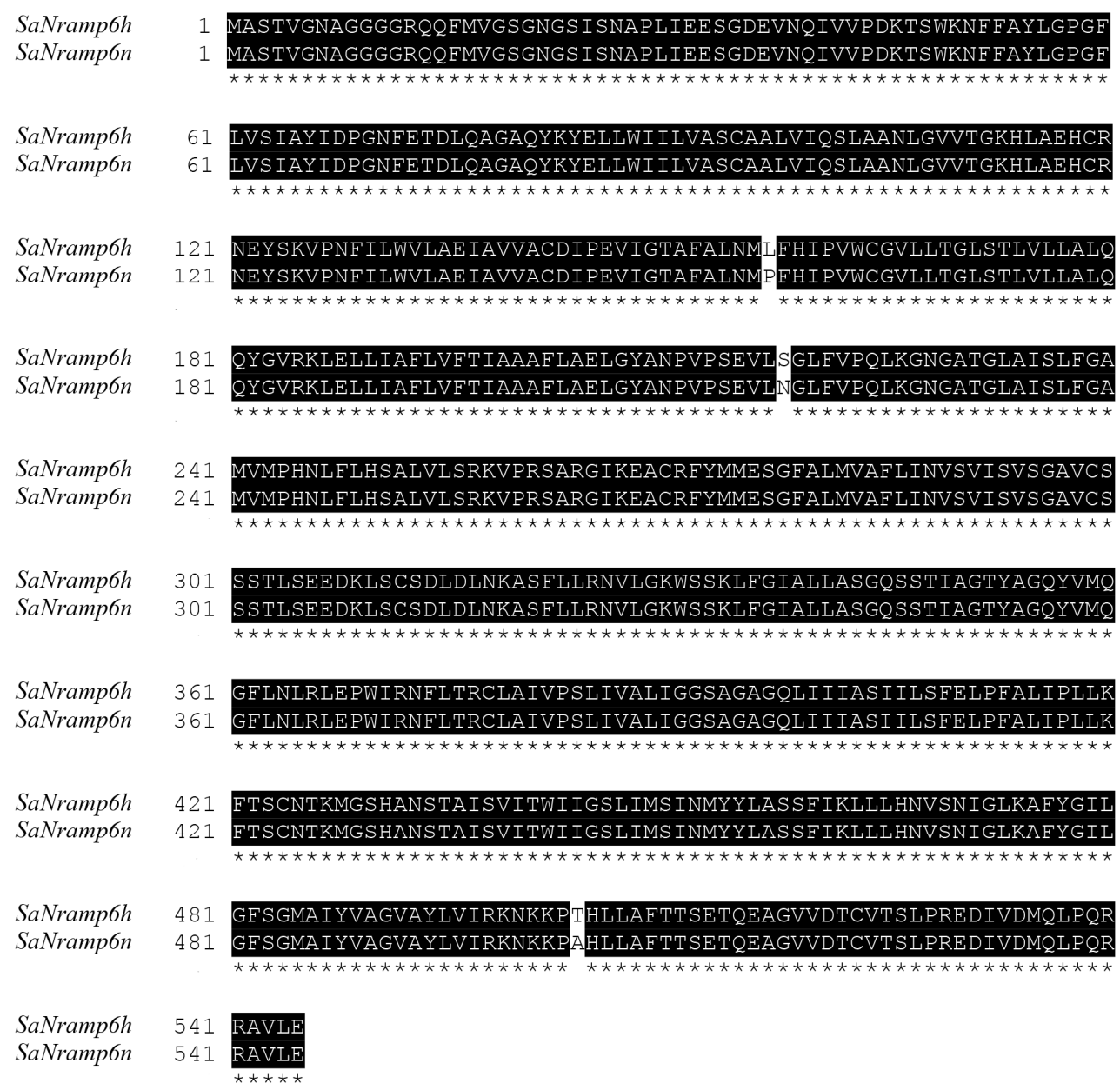

Figure 1. Amino acid sequence alignment of SaNramp6h and SaNramp6n. Sequence alignment was performed by ClustalX. Identical residues are in black and indicated symbol * at the bottom.

The transcript levels of the SaNramp6 genes were compared between the two ecotypes using qRT-PCR analyses. There were no obvious differences in the transcript levels of SaNramp6 in response to $\mathrm{Cd}$ treatment in either ecotype. The transcript levels were higher in shoots and lower in the roots in HE plants compared with the NHE plants. In HE plants, SaNramp6 was primarily expressed in the leaves (Figure 2).

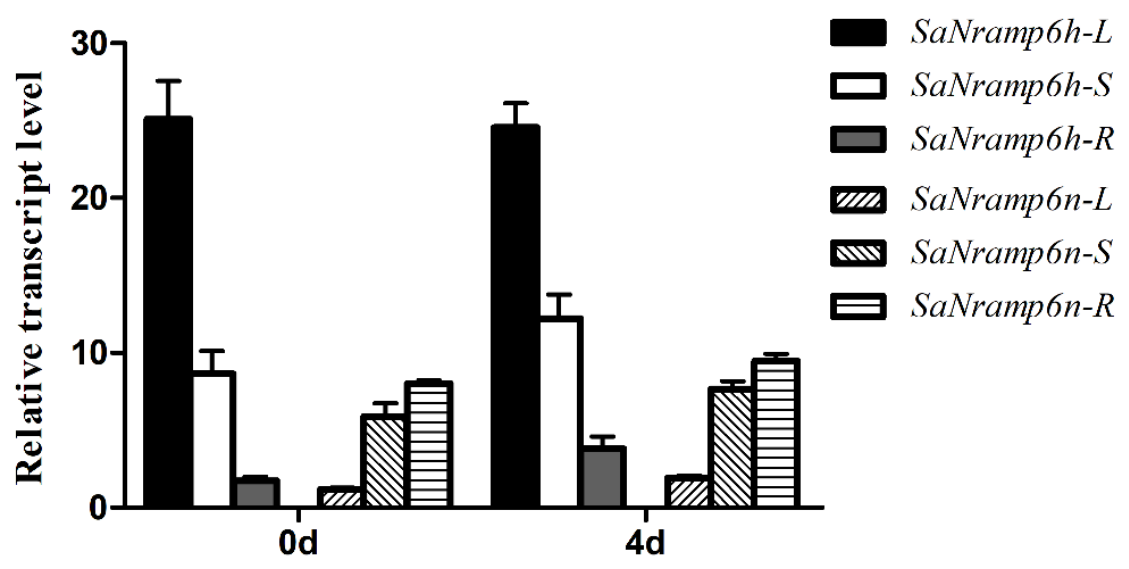

Figure 2. Expression patterns of SaNramp6h and SaNramp6n before and after treatment with cadmium (Cd). L, leaf; S, stem; R, root. Data are means \pm SD ( $n=9$ replicates). 


\subsection{Functional Characterization of Nramp6 in ycf1 Yeast Mutant}

The sequence comparison showed that the vast majority of residues are conserved between SaNramp6h and SaNramp6n, suggesting that they are derived from a common ancestor, and raising the possibility that the residues differing between the two alleles represent candidate sites of functional divergence. The three SaNramp6h-specific residues (Nr1, Nr2, Nr3) were each substituted with the corresponding residues in SaNramp6n (Nr4, Nr5, Nr6) by PCR site-directed mutagenesis. The Cd transport activity of the resulting mutants was tested in a yeast mutant. YCF1 (Yeast cadmium factor 1) is a member of the ABC transporter (ATP-binding cassette transporter) family, and is localized in the tonoplast. It is responsible for the transport of $\mathrm{Cd}$ ions to vacuoles and their detoxification by chelation with glutathione $[25,26]$. Therefore, the $y c f 1$ yeast mutant is sensitive to $\mathrm{Cd}$ at a certain concentration, making it a useful tool for identifying residues involved in Cd transport by SaNramp6. Heterologous expression of either native or mutant SaNramp6 in yeast showed that both SaNramp6h and SaNramp6n could enhance the sensitivity of transgenic yeast to $\mathrm{Cd}$, compared with that of the strain expressing the empty vector. However, ycf1 transformed with SaNramp6n exhibited a smaller degree of reduced growth (Figure 3). The S258N and T504A mutations did not affect the yeast's sensitivity to $\mathrm{Cd}$, but the L157P mutation in SaNramp6h (Nr1) led to a significantly decreased sensitivity to Cd. Thus, when the first amino acid residue of SaNramp6n was replaced with that of SaNramp6h, its phenotype became consistent with that of SaNramp6h, whereas there was no phenotypic difference resulting from the mutations at the other two residues. These results demonstrate the importance of Leu at positon 157 in the $\mathrm{Cd}$ transport activity of the protein.

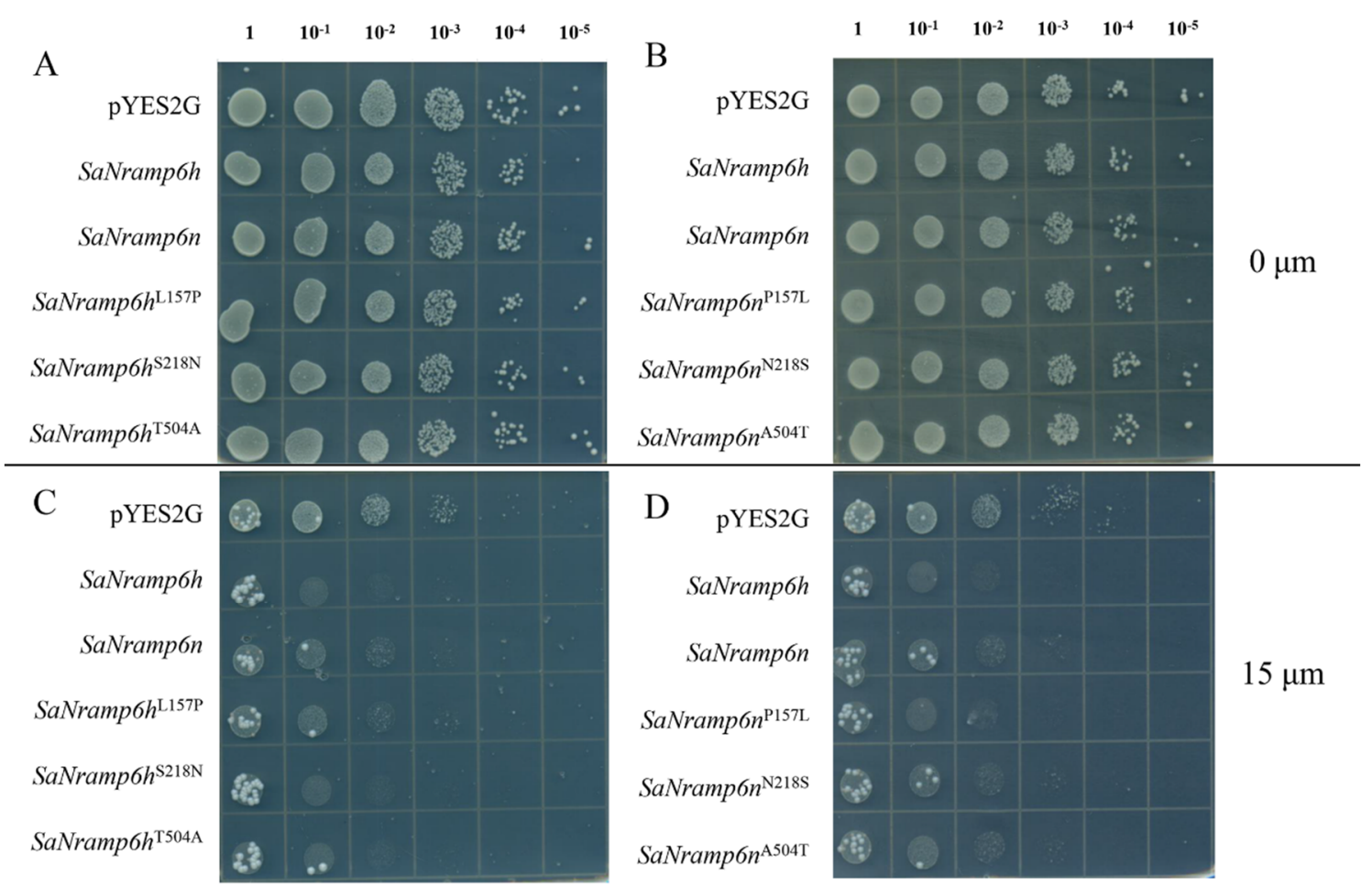

Figure 3. Metal toxicity growth assays of mutated NRAMP6 variants in Saccharomyces cerevisiae mutant ycf1. Yeast strain was transformed with EV (pYES2G empty vector) and other different mutations, and then grown on plates containing synthetic galactose-uracil (SG-U) without $(\mathbf{A}, \mathbf{B})$ or with $15 \mu \mathrm{M}$ $\mathrm{CdCl}_{2}(\mathbf{C}, \mathbf{D})$ for 3 days.

To confirm the involvement of residue 157 in Cd transport, we tested the ability of yeast strains expressing the mutant proteins to grow in the presence of $15 \mu \mathrm{M} \mathrm{CdCl}_{2}$. The strain expressing SaNramp6 showed lower growth rates than that of the negative control, consistent with the results of the spotting assay. Yeast cultures expressing mutated SaNramp6h ${ }^{\mathrm{L} 157 \mathrm{P}}$ exhibited similar or enhanced 
growth rates relative to that of its native counterpart. In contrast, the $\mathrm{P}$ to $\mathrm{L}$ mutation at position 157 in SaNramp6n showed significantly impaired growth (Figure 4). To further evaluate the effect of the mutations, we selected a period of logarithmic growth to measure the metal content in culture cells where results were consistent with the conclusions above (Supplementary Materials Figure S1).

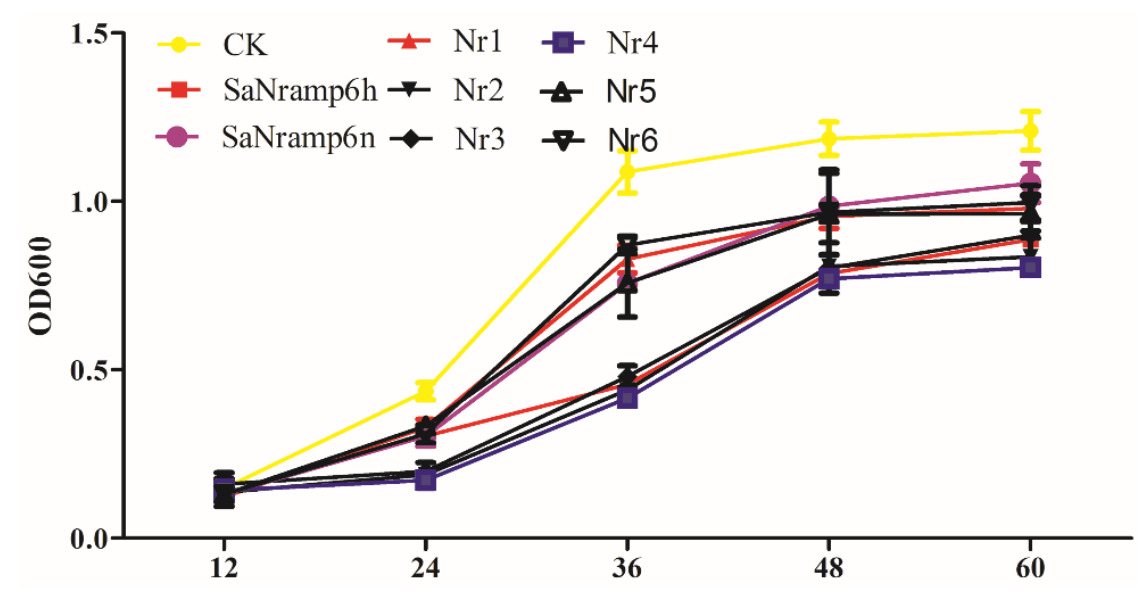

Figure 4. Growth curve of transgenic yeast under cadmium stress. Time-dependent growth of yeast strains in SG-U liquid medium supplemented with $5 \mu \mathrm{M} \mathrm{CdCl}_{2}$.

\subsection{Phenotypic Analysis of Different Transgenic A. thaliana Lines}

To explore the biological relevance of the SaNramp6 mutations in plants, A. thaliana seedlings of wild type (WT) and lines overexpressing SaNramp6h, SaNramp6n, and the mutations at Nr1, Nr2, $\mathrm{Nr} 3, \mathrm{Nr} 4, \mathrm{Nr} 5$, and Nr6 were germinated on $\frac{1}{2}$-strength Murashig and Skoog $\left(\frac{1}{2} \mathrm{MS}\right)$ medium and then transferred to fresh medium with or without $50 \mu \mathrm{M} \mathrm{CdCl}_{2}$. Relative primary root (PR) growth of the seedlings was compared between the control (no $\mathrm{Cd}$ ) and the $\mathrm{Cd}$-containing medium. The results showed that the relative PR length of transgenic Arabidopsis expressing native SaNramp6 and other variants was significantly inhibited. The reduction in root growth of the lines expressing $\mathrm{Nr} 2$ and Nr3 was similar to that of the line expressing SaNramp6h. The root length of the line expressing Nr1 was greater than that of the line expressing SaNramp6h. The lines expressing Nr5 and Nr6 had the same inhibited root growth response as SaNramp6n, whereas the line expressing Nr4 showed very strong inhibition of root growth (Figure 5). Fresh weight and chlorophyll content behaved in a similar manner (Supplementary Materials Figure S2). These results were consistent with those of the plate-based toxicity assay in yeast. We attributed impaired root growth to toxic intracellular accumulation of Cd.

To further investigate the underlying cause of the pronounced difference in $\mathrm{Cd}$ transport between native SaNramp6 and the L157P mutants, we determined metal concentrations in the Arabidopsis lines expressing SaNramp6 and the various mutations in the presence of $30 \mu \mathrm{M} \mathrm{CdCl}_{2}$. The L157P mutation in $\mathrm{Nr} 1$ impaired Cd transport by SaNramp6h, while the S218N mutation in $\mathrm{Nr} 2$ and the T504A mutation in $\mathrm{Nr} 3$ did not affect the $\mathrm{Cd}$ transport function of the protein. However, the concentrations of other mineral nutrients such as Fe and Mn did not differ between the lines (Supplementary Materials Table S1). These results provided further evidence that Leu 157 is involved in Cd transport. The Cd concentration was higher in the line overexpressing $\mathrm{Nr} 4$ than in the lines overexpressing SaNramp6n, $\mathrm{Nr} 5$, and Nr6 (Figure 6), further demonstrating the importance of Leu 157 in Cd accumulation. 
A

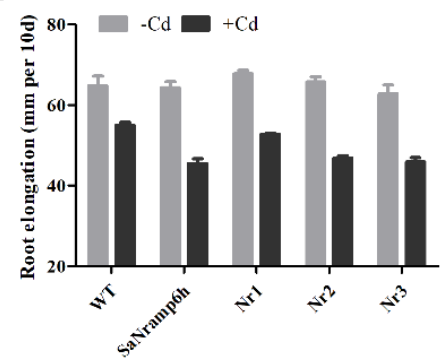

C

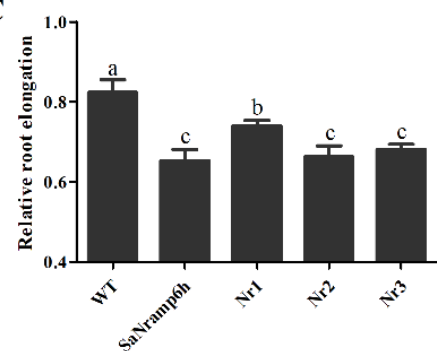

B

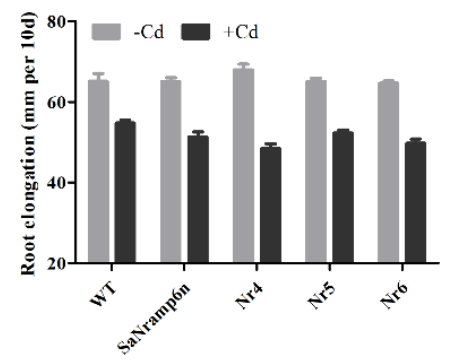

D

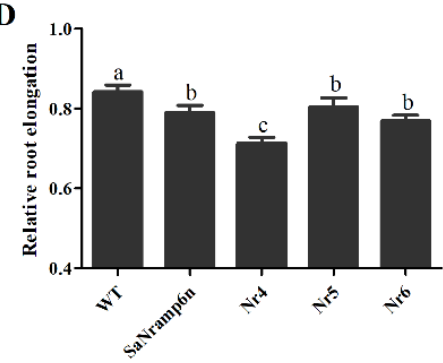

Figure 5. Relative primary root length of transgenic Arabidopsis lines expressing mutations of SaNramp6h. Growth of 14-day-old Arabidopsis Col-0 (wild type) and Arabidopsis lines overexpressing SaNramp6h (from the hyperaccumulating ecotype (HE)), SaNramp6n (from the nonhyperaccumulating ecotype (NHE)), or each of six mutations. Seedlings were germinated on $\frac{1}{2}$ MS medium and transferred to fresh medium supplemented with $50 \mu \mathrm{M} \mathrm{CdCl}_{2}$ for $5 \mathrm{~d}$. (A,B) Primary root length. (C,D) Relative root growth (root growth of seedlings treated with $50 \mu \mathrm{M} \mathrm{CdCl}_{2}$ compared with root growth of untreated seedlings). The level of significance difference is indicated by the letter $a, b, c$. The bars with the same letters are not significantly different at relative root length at $P<0.05$ according to Tukey's test, respectively.

A

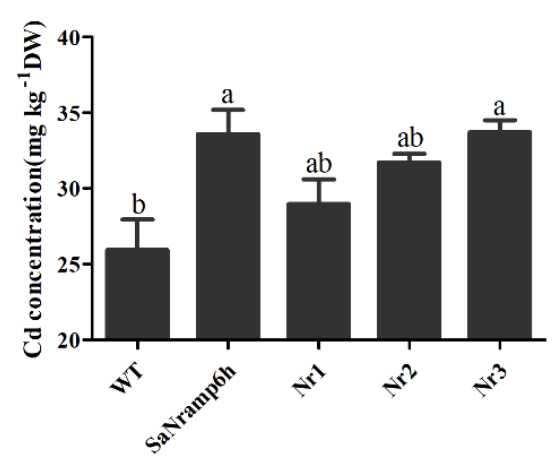

B

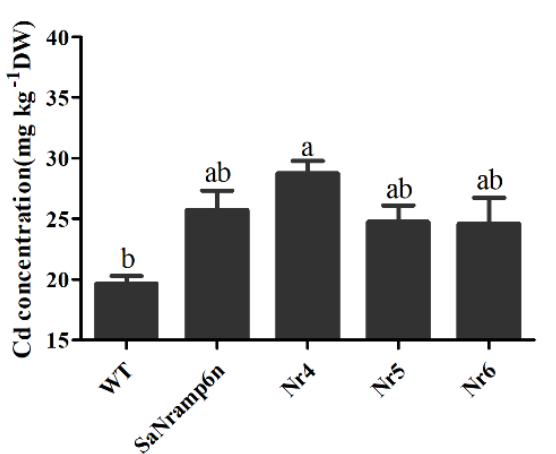

Figure 6. Cadmium (Cd) accumulation in transgenic Arabidopsis lines. Four-week-old hydroponically grown Col-0 (wild type, WT) and lines overexpressing SaNramp6h (from HE), SaNramp6n (from NHE) and six other mutations were transferred to fresh medium containing $30 \mu \mathrm{M} \mathrm{CdCl}_{2}$ and grown for $7 \mathrm{~d}$ before measuring Cd contents. (A) The Cd concentration in WT, SaNramp6h, Nr1, Nr2 and Nr3 mutations. (B) The Cd concentration in WT, SaNramp6n, Nr4, Nr5 and Nr6 mutations.

\section{Discussion}

Genes encoding NRAMPs have been identified in a wide variety of organisms, from bacteria to humans [27]. They play important roles in metal ion homeostasis and are usually highly expressed in hyperaccumulating plants [28]. Previous studies have detailed the selective and molecular transport mechanisms of plant NRAMP proteins for heavy metal ions. Some reports have described enhanced heavy metal transport and accumulation by NRAMP proteins in hyperaccumulator plants. However, the differences in NRAMP proteins between hyperaccumulator plants and nonhyperaccumulator 
plants remain poorly understood. SaNramp6 belongs to the NRAMP family, which is crucial for metal homeostasis, especially for $\mathrm{Fe}, \mathrm{Mn}$, and $\mathrm{Cd}$. Sequence and phylogenetic analyses showed that SaNramp6 contains 11 transmembrane domains and most closely resembles AtNramp6, which is a plasma membrane-localized Cd transporter [17]. In the present study, SaNramp6h and SaNramp6n were found to exhibit different transport activity. SaNramp6h from HE S. alfredii, when expressed in yeast and A. thaliana, could transport Cd more effectively than could SaNramp6n from NHE S. alfredii.

So far, in addition to $S$. alfredii, four other species have been identified as $\mathrm{Cd}$ hyperaccumulators-Arabidopsis halleri [29], Thlaspi praecox [30], Thlaspi caerulescens [31], and Solanum nigrum L. [32]. Compared with nonhyperaccumulating ecotypes, Cd hyperaccumulator plants show enhanced $\mathrm{Cd}$ uptake from soil into root cells, highly efficient $\mathrm{Cd}$ loading into the xylem, enhanced $\mathrm{Cd}$ transport from the roots to shoots, and stronger detoxification of $\mathrm{Cd}$ in the aboveground area [33].

Generally, the absorption of $\mathrm{Cd}$ by higher plants is mainly through the competitive entry via transporters of divalent cations such as $\mathrm{Ca}, \mathrm{Zn}, \mathrm{Fe}$, and $\mathrm{Mn}$. In the hyperaccumulator plants A. halleri and T. praecox, an increase in the exogenous $\mathrm{Zn}$ concentration can reduce $\mathrm{Cd}$ uptake, indicating that $\mathrm{Cd}$ transport depends on a $\mathrm{Zn}$ transporter that has a higher affinity for $\mathrm{Zn}$ than for Cd [34]. However, in N. caerulescens, the hyperaccumulating ecotype (Ganges) may have a high affinity transport system for $\mathrm{Cd}$ that is independent of $\mathrm{Zn}$ transport, because its $\mathrm{Cd}$ uptake is not affected by exogenous $\mathrm{Zn}$ [35]. It has been demonstrated that hyperaccumulators evolve from nonhyperaccumulators under long-term high-level heavy metal conditions [36,37]. During this evolutionary process, transporters may gradually change in their ability to transport metals such as $\mathrm{Cd}$, $\mathrm{Zn}$, and $\mathrm{Pb}$. Heterologous expression of SaHMA3 in S. cerevisiae and tobacco showed that SaHMA3h from HE S. alfredii can only transport Cd, while SaHMA3n from NHE S. alfredii has both Cd and Zn transport activity [38]. In N. caerulescens, NcHMA3 is a specific Cd transporter, but it shows different affinity for $\mathrm{Cd}$ among different genotypes [33]. It is interesting that these homologous genes from different plants have evolved a similar ability to enhance transport activity.

Transporter proteins encoded by different alleles of the gene in different plant genotypes can exhibit different substrate affinity. This provides a useful tool for identifying critical residues for metal transport activity and selectivity. Most residues are conserved so that protein maintains its activity, but some residues responsible for ion selectivity can change without altering protein function. In Arabidopsis AtNRAMP3, the M248A mutant retains Mn transport activity but showed severely reduced Cd transport activity, which decreases Cd sensitivity [39]. Similarly, some mutations in AtNRAMP4 selectively modify $\mathrm{Cd}^{2+}$ and $\mathrm{Zn}^{2+}$ accumulation without affecting Fe transport [40]. In our study, both SaNramp6h and SaNramp6n showed some similarities in function but differed in their $\mathrm{Cd}$ selectivity and expression patterns. In SaNramp6h, the Cd transport activity was significantly decreased by the L157P mutation, but increased by the P157L mutation. However, the S218N and T504A mutations did not affect its transport activity. This indicates that during long-term evolution, residue L157 in SaNramp6h has become responsible for its specific affinity to Cd. Proline is most often thought of as the most rigid residue because of its rigid pyrrolidine ring, and introduction of proline can produce a protein conformational change. Proline substitution at position 157 would enhance the stability of SaNramp6 protein. However, the transport activity of the L157P mutant is decreased relative to that of native SaNramp6h. We infer that L157P might have influenced Cd binding sites of SaNramp6. The discordance between transport activity and stability of the protein needs to be further studied.

The expression pattern of SaNramp6 differed significantly between the HE and NHE plants. The transcript levels of SaNramp6 were significantly higher in HE plants than in NHE plants. The SaNramp6 was constitutively expressed in the shoots. In NHE plants, the transcript levels of SaNramp 6 were slightly higher in the roots than in the shoots. Similar results have been reported for the hyperaccumulating ecotype (Ganges) of N. caerulescens, based on a comparison of the transcriptomes between two ecotypes. Further research showed that NcNramp1 participates in the transport of Cd into the stele and from the root to shoot. Increased expression of several genes encoding metal transporters 
was identified as the main reason for Cd hyperaccumulation in N. caerulescens [41]. In the case of the expression of Nramp6 under the 35S promoter, such as in the present study, all the plant organs accumulated $\mathrm{Cd}$ and significant sensitization might be expected because of incorrect tissue specificity. However, in the HE plants, Nramp6 seems to function as an enhancer for improved shoot internal sequestration in combination with the buffering of physiologically available $\mathrm{Cd}$ in the cytosol or efflux into the vacuole of the shoot cell. Thus, our data highlight two aspects of the roles of SaNramp 6 in Cd sequestration-elevated transcript levels in the shoots may be associated with high $\mathrm{Cd}$ accumulation in shoots, and lower expression levels in the roots may explain why less $\mathrm{Cd}$ is retained in the roots.

\section{Materials and Methods}

\subsection{Yeast Strain and Culture Conditions}

The Cd-sensitive S. cerevisiae strain BY4742 $\Delta y$ cf1 (MAT $\alpha$; his $3 \Delta 1$; leu2 $\Delta 0$; met15 $\Delta 0$; ura $3 \Delta 0$; YDR135c::kanMX4) was used in this study. Yeast cells were transformed by electroporation [42]. Positive transformants were selected on synthetic defined medium lacking uracil (SD-U), containing $2 \%(w / v)$ glucose.

\subsection{Gene Cloning and Expression Analysis}

Total RNA was extracted from root, stem and leaf tissues of $S$. alfredii seedlings using a Plant Tissue Total RNA Purification Kit (NORGEN, Thorold, ON, Canada) according to the manufacturer's instructions. The reverse transcription reactions were performed using a Superscript RT III first-strand cDNA synthesis kit followed by treatment with RNase H (Invitrogen, Carlsbad, CA, USA). The CDS fragments of SaNramp6n were amplified by high fidelity KOD-Plus DNA Polymerase (Toyobo, Osaka, Japan) using the primer, which were originally used to amplify the SaNramp6h allele [20]. The amino acid sequences of SaNramp6h and SaNramp6n were compared using ClustalX.

To evaluate the effect of $\mathrm{Cd}$ on the expression of SaNramp6, S. alfredii HE and NHE plants were treated with $100 \mu \mathrm{M} \mathrm{CdCl}_{2}$ for 4 days. The plants were divided into leaves, stem, and roots, which were immediately frozen in liquid nitrogen before extracting RNA. The SaNramp6 expression levels were quantified using PrimeScript ${ }^{\mathrm{TM}}$ RT SYBR Green Master Mix (Takara, Dalian, China). The transcript levels were calculated relative to that of SaUBC9 as the reference gene [20].

\subsection{Site-Directed Mutagenesis and Construction of Expression Vector}

The cDNAs of SaNramp6h and SaNramp6n were reciprocally mutagenized by a PCR-mediated site-directed mutation technique [43]. First, single mutants were introduced into the primers B1 and B2. To facilitate the amplification of the target fragment, we added the entry vector connector sequence before the full-length primer. The upper half of the target fragment was amplified using the full-length upstream primer A1 and the downstream primer B1, and then the lower half of the target fragment was amplified by the full-length downstream primer A2 and the upstream primer B2. Finally, the full-length primers A1 and A2 and the products recovered from the upper and lower segments were used as templates to amplify the complete target fragments. The purified full-length PCR fragment was inserted into the Gateway entry vector pENTR/D-Topo (Invitrogen, Carlsbad, CA, USA), then LR-recombined into the yeast expression vector pYES2.0 or the plant expression vector pH2GW7.0 [44]. Recombinant plasmids carrying native and mutated SaNramp6h and SaNramp6n were introduced into the yeast strain. The empty plasmid pYES2G was transformed into yeast cells as the negative control. Diluted cultures of individual transformants were spotted onto synthetic galactose-uracil (SG-U) agar plates containing 0 or $15 \mu \mathrm{M} \mathrm{CdCl}_{2}$. The plates were incubated at $30^{\circ} \mathrm{C}$ for 3 days before growth phenotypes were evaluated. The yeast strains were also grown overnight in $10 \mathrm{~mL}$ minimal medium and then transferred to $100 \mathrm{~mL} \mathrm{SG}-\mathrm{U}$ containing $5 \mu \mathrm{M} \mathrm{CdCl}_{2}$, and the relative growth of the transformants was determined by measuring the $\mathrm{OD}_{600}$ every $6 \mathrm{~h}$. The yeast cells were harvested for $36 \mathrm{~h}$ and washed with PBS before being dried and digested with a concentrated acid 
mixture of $\mathrm{HNO} 3, \mathrm{HClO} 4$, and $\mathrm{H} 2 \mathrm{SO} 4$ (volume ratio $=4: 1: 0.5$ ) at $250{ }^{\circ} \mathrm{C}$ for $8 \mathrm{~h}$. The determination of Cd content via Inductively Coupled Plasma-Mass Spectrometry (ICP-MS) (NexION 300; PerkinElmer, Shelton, CT, USA).

\subsection{Plant Materials and Growth Conditions}

The HE S. alfredii plants originated from an old $\mathrm{Pb} / \mathrm{Zn}$ mining region near the city of Quzhou in Zhejiang province, P. R. China. The NHE plants were obtained from a tea plantation in Hangzhou, Zhejiang province, P. R. China. The plant materials were cutting clones of the original plants, and were grown in half-strength Hoagland-Arnon solution, at $25^{\circ} \mathrm{C}$ under a $16 \mathrm{hlight} / 8 \mathrm{~h}$ dark photoperiod in an artificial climate incubator. The Columbia ecotype of $A$. thaliana from our laboratory was used for Agrobacterium-mediated transformation with pH2GW7.0-SaNramp6 or SaNramp6 point mutants.

\subsection{Acquisition of Transgenic Arabidopsis and Cadmium Stress Treatment}

The recombinant plasmid pH2GW7.0-SaNramp6 was transformed into Agrobacterium tumefaciens EHA105, and the positive strain was then used to transfect Arabidopsis via the floral dip method [45,46]. Three hygromycin-resistant Arabidopsis lines were obtained after transformation with the SaNramp6 construct. Transgenic lines that were PCR positive (Supplemental Figure S3) with similar expression levels of SaNramp6 (Supplemental Figures S4 and S5) were used for subsequent experiments. Seeds from one homozygous line T3 and the WT were placed on MS agar plates containing $50 \mu \mathrm{M} \mathrm{CdCl}_{2}$ using a sterilized toothpick. After vertical cultivation for 14 days in a greenhouse at $22{ }^{\circ} \mathrm{C}$, roots were scanned using an Epson Perfection V700 Photo scanner (Seiko Epson Corp., Nagano, Japan). The length of roots was determined by WinRHIZO PRO2012a (Regent Instruments Inc., Quebec, Canada), for comparison of root growth among the WT and transgenic lines (Supplemental Figure S6).

The hydroponic system was based on the modified system of Chen et al. (2017) [20]. The seeds of WT and different transgenic $A$. thaliana lines were cultured in $\frac{1}{2}$ MS solid medium for 1 week, and then grown in $1 / 4$ Hoagland's nutrient solution for 14 days under hydroponic conditions. Subsequently, the seedlings were treated with $30 \mathrm{mM} \mathrm{CdCl}_{2}$ for 7 days. All the seedlings were collected and fresh weights were determined. Chlorophyll was extracted by $90 \%$ acetone.

\subsection{Element Content Analysis}

For analyses of metal content in plants, roots and shoots were harvested separately. The roots were chelated with $1 \mathrm{mM}$ EDTA for $30 \mathrm{~min}$ and then rinsed with distilled water three times. The dried samples were ground and subsequently digested in nitric acid. The $\mathrm{Fe}, \mathrm{Mn}$, and $\mathrm{Cd}$ concentration was determined by Inductively Coupled Plasma-Mass Spectrometry (ICP-MS) (NexION 300; PerkinElmer, Shelton, CT, USA).

\section{Conclusions}

SaNramp6 from S. alfredii is a transport protein localized to the plasma membrane. Compared with SaNramp6n, SaNramp6h shows higher affinity for Cd. Amino acid residue L157 was identified as being critical for the $\mathrm{Cd}$ transport activity of SaNramp6h. The differences in the ionic selectivity and expression of the Nramp6 transporters between hyperaccumulating and nonhyperaccumulating ecotypes provide important clues about the mechanisms of $\mathrm{Cd}$ hyperaccumulation and tolerance in the hyperaccumulator S. alfredii. 
Supplementary Materials: The following are available online at http://www.mdpi.com/1422-0067/21/9/3169/s1, Table S1: Elemental concentrations in shoots and roots of wild-type and transgenic Arabidopsis lines expressing mutations of SaNramp6, Figure S1: Cd content of $\Delta$ ycf1 yeast cells expressing mutations of SaNramp6, Figure S2: Biomass analyses and chlorophyll content of wild-type and transgenic Arabidopsis lines expressing mutations of SaNramp6, Figure S3: PCR identification of transgenic Arabidopsis thaliana lines, Figure S4: RT-PCR analysis of the SaNramp6 transcript in the wildtype (WT) and six site mutant mutations plants, Figure S5: Relative expression of SaNramp6 in transgenic Arabidopsis lines expressing mutations of SaNramp6, Figure S6: Phenotypes of wild-type (WT) and overexpressing SaNramp6h, SaNramp6n and other six mutations of Arabidopsis thaliana under Cd stress.

Author Contributions: Z.L. and W.Q. contributed to data analysis and manuscript preparation. R.Z. planned and designed the research. Z.L., S.C., W.Q., X.H., and G.Q. performed the experiments, J.Z., Z.L., W.Q., X.H. wrote the manuscript and coordinated its revision. J.Z., S.C., Y.J. and G.Q. helped in sample preparation and data collection. All authors have read and agreed to the published version of the manuscript.

Funding: This research was funded by grant from the National Nonprofit Institute Research Grant of CAF (CAFYBB2019SZ001), the National key program on transgenic Research (2018ZX08020002) and the National Natural Science Foundation of China (No. 31872168) supported this work.

Acknowledgments: We thank Jennifer Smith, from Liwen Bianji, Edanz Group China (www.liwenbianji.cn/ac), for editing the English text of a draft of this manuscript.

Conflicts of Interest: The authors declare no conflict of interest.

$\begin{array}{ll}\text { Abbreviations } \\ \mathrm{Cd} & \text { Cadmium } \\ \mathrm{Nr} 1 & \text { SaNramp6h } h^{\mathrm{L} 157 \mathrm{P}} \\ \mathrm{Nr} 2 & \text { SaNramp } 6 h^{\mathrm{S} 218 \mathrm{~N}} \\ \mathrm{Nr} 3 & \text { SaNramp6h} h^{\mathrm{T} 504 \mathrm{~A}} \\ \mathrm{Nr} 4 & \text { SaNramp6n } n^{\mathrm{P} 157 \mathrm{~L}} \\ \mathrm{Nr} 5 & \text { SaNramp6n }{ }^{\mathrm{N} 218 \mathrm{~S}} \\ \mathrm{Nr} 6 & \text { SaNramp6n } \\ \mathrm{A} 504 \mathrm{~T} \\ \mathrm{~L} & \text { Leucine } \\ \mathrm{S} & \text { Serine } \\ \mathrm{T} & \text { Threonine } \\ \mathrm{P} & \text { Proline } \\ \mathrm{N} & \text { Asparagine } \\ \mathrm{A} & \text { Alanine }\end{array}$

\section{References}

1. Satarug, S.; Garrett, S.H.; Sens, M.A.; Sens, D.A. Cadmium, Environmental Exposure, and Health Outcomes. Environ. Health Perspect. 2010, 118, 182-190. [CrossRef] [PubMed]

2. Zhao, F.-J.; Ma, Y.; Zhu, Y.-G.; Tang, Z.; McGrath, S.P. Soil Contamination in China: Current Status and Mitigation Strategies. Environ. Sci. Technol. 2015, 49, 750-759. [CrossRef] [PubMed]

3. Brooks, R.R.; Lee, J.; Reeves, R.D.; Jaffré, T. Detection of nickeliferous rocks by analysis of herbarium specimens of indicator plants. J. Geochem. Explor. 1977, 7, 49-57. [CrossRef]

4. Rascio, N.; Navari-Izzo, F. Heavy metal hyperaccumulating plants: How and why do they do it? And what makes them so interesting? Plant Sci. 2011, 180, 169-181. [CrossRef]

5. Montanini, B.; Blaudez, D.; Jeandroz, S.; Sanders, D.; Chalot, M. Phylogenetic and functional analysis of the Cation Diffusion Facilitator (CDF) family: Improved signature and prediction of substrate specificity. BMC Genom. 2007, 8, 107. [CrossRef]

6. Wong, C.K.E.; Cobbett, C.S. HMA P-type ATPases are the major mechanism for root-to-shoot Cd translocation in Arabidopsis thaliana. New Phytol. 2009, 181, 71-78. [CrossRef]

7. Curie, C.; Cassin, G.; Couch, D.; Divol, F.; Higuchi, K.; Le Jean, M.; Misson, J.; Schikora, A.; Czernic, P.; Mari, S. Metal movement within the plant: Contribution of nicotianamine and yellow stripe 1-like transporters. Ann. Bot. 2008, 103, 1-11. [CrossRef]

8. Waters, B.M.; Chu, H.-H.; DiDonato, R.J.; Roberts, L.A.; Eisley, R.B.; Lahner, B.; Salt, D.E.; Walker, E.L. Mutations in Arabidopsis yellow stripe-like1 and yellow stripe-like3 reveal their roles in metal ion homeostasis and loading of metal ions in seeds. Plant Physiol. 2006, 141, 1446-1458. [CrossRef] 
9. Park, J.; Song, W.Y.; Ko, D.; Eom, Y.; Hansen, T.H.; Schiller, M.; Lee, T.G.; Martinoia, E.; Lee, Y. The phytochelatin transporters AtABCC1 and AtABCC2 mediate tolerance to cadmium and mercury. Plant J. 2012, 69, 278-288. [CrossRef]

10. Yokosho, K.; Yamaji, N.; Ueno, D.; Mitani, N.; Ma, J.F. OsFRDL1 is a citrate transporter required for efficient translocation of iron in rice. Plant Physiol. 2009, 149, 297-305. [CrossRef]

11. Pittman, J.; Hirschi, K. CAX-ing a wide net: Cation/H+ transporters in metal remediation and abiotic stress signalling. Plant Physiol. 2016, 18, 741-749. [CrossRef] [PubMed]

12. Guerinot, M.L. The ZIP family of metal transporters. Biochim. Biophys. Acta (BBA)-Biomembr. 2000, 1465, 190-198. [CrossRef]

13. Sasaki, A.; Yamaji, N.; Yokosho, K.; Ma, J.F. Nramp5 Is a Major Transporter Responsible for Manganese and Cadmium Uptake in Rice. Plant Cell 2012, 24, 2155-2167. [CrossRef] [PubMed]

14. Yang, Z.; Chu, C. Towards understanding plant response to heavy metal stress. In Abiotic Stress in Plants-Mechanisms and Adaptations; InTech: Rijeka, Croatia, 2011; pp. 59-78.

15. Curie, C.; Alonso, J.M.; Marie, L.; Ecker, J.R.; Briat, J.-F. Involvement of NRAMP1 from Arabidopsis thaliana in iron transport. Biochem. J. 2000, 347, 749-755. [CrossRef] [PubMed]

16. Lanquar, V.; Lelievre, F.; Barbier-Brygoo, H.; Thomine, S. Regulation and function of AtNRAMP4 metal transporter protein. Soil Sci. Plant Nutr. 2004, 50, 1141-1150. [CrossRef]

17. Cailliatte, R.; Lapeyre, B.; Briat, J.-F.; Mari, S.; Curie, C. The NRAMP6 metal transporter contributes to cadmium toxicity. Biochem. J. 2009, 422, 217-228. [CrossRef]

18. Takahashi, R.; Ishimaru, Y.; Shimo, H.; Bashir, K.; Senoura, T.; Sugimoto, K.; Ono, K.; Suzui, N.; Kawachi, N.; Ishii, S.; et al. From Laboratory to Field: OsNRAMP5-Knockdown Rice is a Promising Candidate for Cd Phytoremediation in Paddy Fields. PLoS ONE 2014, 9, e98816. [CrossRef]

19. Zhang, J. Molecular Mechanisms of Cd Tolerance and Accumulation in Metal Hyperaccumulator Sedum Alfredii; Zhejiang University: Hangzhou, China, 2015.

20. Chen, S.; Han, X.; Fang, J.; Lu, Z.; Qiu, W.; Liu, M.; Sang, J.; Jiang, J.; Zhuo, R. Sedum alfredii SaNramp6 Metal Transporter Contributes to Cadmium Accumulation in Transgenic Arabidopsis thaliana. Sci. Rep. 2017, 7, 13318. [CrossRef]

21. Yang, X.; Long, X.X.; Ni, W.Z.; Fu, C.X. Sedum alfredii H: A new Zn hyperaccumulating plant first found in China. Chin. Sci. Bull. 2002, 47, 1634-1637. [CrossRef]

22. Lu, L.-L.; Tian, S.-K.; Yang, X.-E.; Wang, X.-C.; Brown, P.; Li, T.-Q.; He, Z.-L. Enhanced root-to-shoot translocation of cadmium in the hyperaccumulating ecotype of Sedum alfredii. J. Exp. Bot. 2008, 59, 3203-3213. [CrossRef]

23. Yang, X.E.; Long, X.X.; Ye, H.B.; He, Z.L.; Calvert, D.V.; Stoffella, P.J. Cadmium tolerance and hyperaccumulation in a new Zn-hyperaccumulating plant species (Sedum alfredii Hance). Plant Soil 2004, 259, 181-189. [CrossRef]

24. Yang, Q.; Shohag, M.J.I.; Feng, Y.; He, Z.; Yang, X. Transcriptome Comparison Reveals the Adaptive Evolution of Two Contrasting Ecotypes of Zn/Cd Hyperaccumulator Sedum alfredii Hance. Front. Plant Sci. 2017, 8, 343. [CrossRef] [PubMed]

25. Li, Z.-S.; Lu, Y.-P.; Zhen, R.-G.; Szczypka, M.; Thiele, D.J.; Rea, P.A. A new pathway for vacuolar cadmium sequestration in Saccharomyces cerevisiae: YCF1-catalyzed transport of bis (glutathionato) cadmium. Proc. Natl. Acad. Sci. USA 1997, 94, 42-47. [CrossRef] [PubMed]

26. Preveral, S.; Ansoborlo, E.; Mari, S.; Vavasseur, A.; Forestier, C. Metal (loid) s and radionuclides cytotoxicity in Saccharomyces cerevisiae. Role of YCF1, glutathione and effect of buthionine sulfoximine. Biochimie 2006, 88, 1651-1663. [CrossRef]

27. Nevo, Y.; Nelson, N. The NRAMP family of metal-ion transporters. Biochim. Biophys. Acta (BBA)-Bioenerg. 2006, 1763, 609-620. [CrossRef]

28. Oomen, R.J.F.J.; Wu, J.; Lelievre, F.; Blanchet, S.; Richaud, P.; Barbier-Brygoo, H.; Aarts, M.G.M.; Thomine, S. Functional characterization of NRAMP3 and NRAMP4 from the metal hyperaccumulator Thlaspi caerulescens. New Phytol. 2008, 181, 637-650. [CrossRef]

29. Dahmani-Muller, H.; Van Oort, F.; Gelie, B.; Balabane, M. Strategies of heavy metal uptake by three plant species growing near a metal smelter. Environ. Pollut. 2000, 109, 231-238. [CrossRef] 
30. McGrath, S.; Sidoli, C.; Baker, A.; Reeves, R. The potential for the use of metal-accumulating plants for the in situ decontamination of metal-polluted soils. In Integrated Soil and Sediment Research: A Basis for Proper Protection; Soil \& Environment; Springer: Dordrecht, The Netherlands, 1993; Volume 1, pp. 673-676.

31. Lombi, E.; Zhao, F.; Dunham, S.; McGrath, S. Cadmium accumulation in populations of Thlaspi caerulescens and Thlaspi goesingense. New Phytol. 2000, 145, 11-20. [CrossRef]

32. Deng, X.; Xia, Y.; Hu, W.; Zhang, H.; Shen, Z. Cadmium-induced oxidative damage and protective effects of N-acetyl-1-cysteine against cadmium toxicity in Solanum nigrum L. J. Hazard. Mater. 2010, 180, 722-729. [CrossRef]

33. Ueno, D.; Milner, M.J.; Yamaji, N.; Yokosho, K.; Koyama, E.; Clemencia Zambrano, M.; Kaskie, M.; Ebbs, S.; Kochian, L.V.; Ma, J.F. Elevated expression of TcHMA3 plays a key role in the extreme Cd tolerance in a Cd-hyperaccumulating ecotype of Thlaspi caerulescens. Plant J. 2011, 66, 852-862. [CrossRef]

34. Zhao, F.J.; Hamon, R.E.; Lombi, E.; McLaughlin, M.J.; McGrath, S.P. Characteristics of cadmium uptake in two contrasting ecotypes of the hyperaccumulator Thlaspi caerulescens. J. Exp. Bot. 2002, 53, 535-543. [CrossRef]

35. Lombi, E.; Zhao, F.; McGrath, S.; Young, S.; Sacchi, G. Physiological evidence for a high-affinity cadmium transporter highly expressed in a Thlaspi caerulescens ecotype. New Phytol. 2001, 149, 53-60. [CrossRef]

36. Cappa, J.J.; Pilon-Smits, E.A.H. Evolutionary aspects of elemental hyperaccumulation. Planta 2014, 239, 267-275. [CrossRef]

37. Krämer, U. Metal hyperaccumulation in plants. Annu. Rev. Plant Biol. 2010, 61, 517-534. [CrossRef]

38. Zhang, J.; Zhang, M.; Shohag, M.J.I.; Tian, S.; Song, H.; Feng, Y.; Yang, X. Enhanced expression of SaHMA3 plays critical roles in Cd hyperaccumulation and hypertolerance in Cd hyperaccumulator Sedum alfredii Hance. Planta 2013, 239, 267-275. [CrossRef]

39. Li, J.; Wang, L.; Zheng, L.; Wang, Y.; Chen, X.; Zhang, W. A Functional Study Identifying Critical Residues Involving Metal Transport Activity and Selectivity in Natural Resistance-Associated Macrophage Protein 3 in Arabidopsis thaliana. Int. J. Mol. Sci. 2018, 19, 1430. [CrossRef]

40. Pottier, M.; Oomen, R.; Picco, C.; Giraudat, J.; Scholz-Starke, J.; Richaud, P.; Carpaneto, A.; Thomine, S. Identification of mutations allowing Natural Resistance Associated Macrophage Proteins (NRAMP) to discriminate against cadmium. Plant J. 2015, 83, 625-637. [CrossRef]

41. Milner, M.J.; Mitani-Ueno, N.; Yamaji, N.; Yokosho, K.; Craft, E.; Fei, Z.; Ebbs, S.; Zambrano, M.C.; Ma, J.F.; Kochian, L.V. Root and shoot transcriptome analysis of two ecotypes of Noccaea caerulescens uncovers the role of NcNramp1 in Cd hyperaccumulation. Plant J. 2014, 78, 398-410. [CrossRef]

42. Meilhoc, E.; Masson, J.-M.; Teissié, J. High efficiency transformation of intact yeast cells by electric field pulses. Bio/technology 1990, 8, 223-227. [CrossRef]

43. Heckman, K.L.; Pease, L.R. Gene splicing and mutagenesis by PCR-driven overlap extension. Nat. Protoc. 2007, 2, 924-932. [CrossRef]

44. Karimi, M.; Inzé, D.; Depicker, A. GATEWAYTM vectors for Agrobacterium-mediated plant transformation. Trends Plant Sci. 2002, 7, 193-195. [CrossRef]

45. Bechtold, N.; Bouchez, D. In planta Agrobacterium-mediated transformation of adult Arabidopsis thaliana plants by vacuum infiltration. In Gene Transfer to Plants; Methods in Molecular Biology ${ }^{\mathrm{TM}}$; Humana Press: Totowa, NJ, USA, 1995; Volume 82, pp. 19-23.

46. Clough, S.J.; Bent, A.F. Floral dip: A simplified method for Agrobacterium-mediated transformation of Arabidopsis thaliana. Plant J. 1998, 16, 735-743. [CrossRef]

(C) 2020 by the authors. Licensee MDPI, Basel, Switzerland. This article is an open access article distributed under the terms and conditions of the Creative Commons Attribution (CC BY) license (http://creativecommons.org/licenses/by/4.0/). 